\title{
Pick_Sw: A Program for Interactive Picking of S-Wave Data, Version 2.00
}

by Karl J. Ellefsen ${ }^{1}$

Open-File Report 02-176

2002

This report is preliminary and has not been reviewed for conformity with U.S. Geological Survey editorial standards. Any use of trade, product, or firm names is for descriptive purposes only and does not imply endorsement by the U.S. Government.

\section{U.S. DEPARTMENT OF THE INTERIOR}

U.S. GEOLOGICAL SURVEY

${ }^{1}$ Denver, Colorado 


\section{OVERVIEW}

Program pick_sw is used to interactively pick travel times from S-wave data. It is assumed that the data are collected using 2 shots of opposite polarity at each shot location. The traces must be in either the SEG-2 format or the SU format. The program is written in the IDL and $\mathrm{C}$ programming languages, and the program is executed under the Windows operating system. (The program may also execute under other operating systems like UNIX if the C language functions are re-compiled).

This Open-File Report describes

- The contents and organization of the Zip file (section 2).

- Program pick_sw, including the program files, the installation of the program, the input files, and the execution of the program (section 3).

- Test data and some plots of the data (section 4).

- References having extensive information about the SEG-2 and the SU data formats (section 5).

- The address of the distributor of the IDL programming language (section 6).

- Addresses to which suggestions for improving the program may be sent (section 7).

The first release of pick_sw was version 1.00. The program was extensively revised and is now released as version 2.00. Compared to the previous version, this new version is easier to install, has GUIs that are easier to use, and has GUIs offering more capabilities. ("GUI" means graphical user interface.) In addition, the new version is object-oriented, making it easier to maintain.

\section{CONTENTS AND ORGANIZATION OF THE ZIP FILE}

The name of the Zip file is "pick_sw.zip." Within the Zip file are ofr_02-176.pdf, 18 files, and a directory labeled "test_data." File ofr_02-176.pdf is a copy of this file. The 18 files are needed to execute the program and are described in section 3.1. Directory test_data has 9 files that are either input to or output from program pick_sw. All 9 files are $\overline{\text { described in section } 4 .}$

The contents of pick_sw.zip may be extracted using program Winzip, which may be purchased at http://www.winzip.com.

\section{PROGRAM Pick_Sw}

\subsection{Files}

The computer code for program pick_sw is in 18 different files, which are briefly described in Table 1. 
Table 1. Files with the code for program pick_sw.

\begin{tabular}{|l|l|}
\hline File & Short Description \\
\hline pick_sw.prj & Project file used by IDL. \\
\hline pick_sw.pro & Creates the GUIs. \\
\hline pick_sw_define.pro & Reads, processes, and plots the traces and the travel times. \\
\hline error_message.pro & Prints an error message on the screen. \\
\hline lib_name.pro & Determines the appropriate extension of a filename for a library. \\
\hline handle_error.pro & Handles an error within an IDL procedure. (include file) \\
\hline handle_error_gr.pro & Handles an error within an IDL procedure that creates graphics. (include file) \\
\hline handle_error_rw.pro & $\begin{array}{l}\text { Handles an error within an IDL procedure that reads and writes files. } \\
\text { (include file) }\end{array}$ \\
\hline About.txt & ASCII text file with general information about program pick_sw. \\
\hline Help.txt & ASCII text file with information about the main GUI of program pick_sw. \\
\hline Help_traces.txt & $\begin{array}{l}\text { ASCII text file with information about the GUI used to view the traces, pick } \\
\text { the travel times, and assign weights. }\end{array}$ \\
\hline Help_spread.txt & ASCII text file with information about the GUI used to view the spread. \\
\hline Help_times.txt & ASCII text file with information about the GUI used to view the travel times. \\
\hline fnc_pick_sw.c & $\begin{array}{l}\text { C-language functions used to read traces in the SEG-2 and the SU data } \\
\text { formats. }\end{array}$ \\
\hline fnc_pick_sw.def & ASCII file needed by the Visual C++ compiler. \\
\hline su_head.h & C-language header used to define the trace header for the SU data format. \\
\hline seg2.h & C-language header used to define the trace header for the SEG-2 data format. \\
\hline fnc_pick_sw.dll & Function fnc_pick_sw.c compiled as a dynamic link library. \\
\hline
\end{tabular}

\subsection{Installation}

The installation requires three steps:

1. Copy the Zip file to a suitable directory on your computer.

2. Extract the contents of the Zip file using WinZip.

3. Load procedure pick sw.pro into an editor and go to line 1587, which is ext_dir $=$ 'd: $\backslash$ seismic $\backslash$ processing $\backslash$ pick_sw $\backslash$ fnc_pick_sw $\backslash$ debug $\backslash '$

Variable ext_dir is a string with the path to the library fnc_pick_sw.dll. Set this variable to the correct path. Be sure to include the quotes at the beginning and the end of the string.

\subsection{Input Data}

\subsubsection{Information File}

This file contains information about the receiver locations, the shot locations, the pairing of the shots with opposite polarities, and so on. The format of this file is described using "spr_info.txt," which is an example of an information file and is located in directory test_data: 


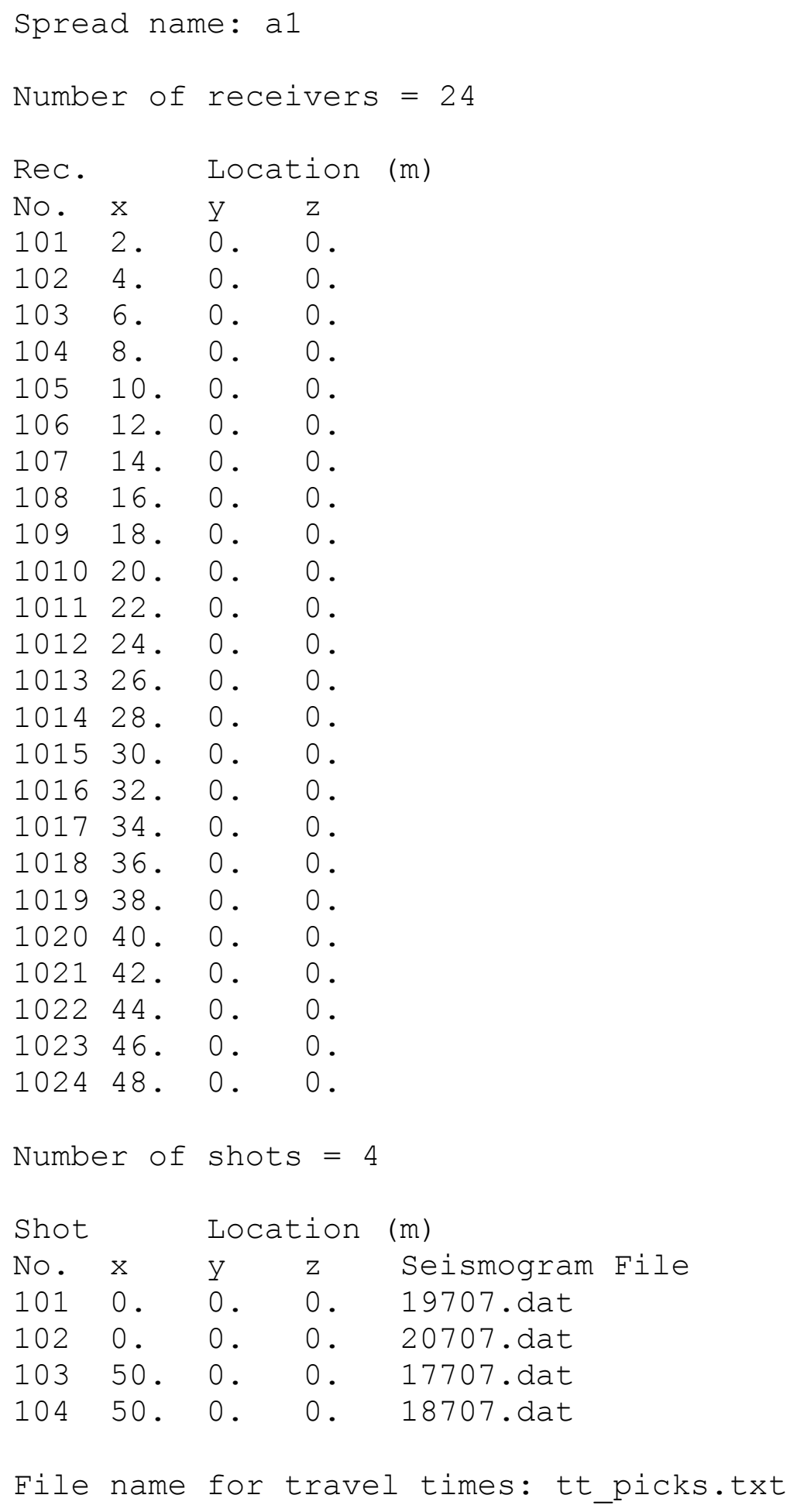

Because all entries in this file are labeled, only a few remarks are necessary.

The number of shots must be even because the $S$-wave data are assumed to be collected with both polarities of the source. The polarities are indicated by the order of the shots in the information file: Shots 1 and 2 have the same location but different polarities; 
likewise, shots 3 and 4 have the same location but different polarities; and so on. The program checks that shots 1 and 2 have the same location, that shots 3 and 4 have the same location, and so on.

The coordinate system used to specify the locations of the receivers and the shots does not affect the picking of the travel times. However, the coordinate system does affect the plots of the spread and the traveltimes (sections 4.4 and 4.5, respectively). The coordinate system should be oriented such that the $x$ axis is parallel to the spread, the $y$ axis is perpendicular to the spread, and the $z$ axis is vertical. Thus, for a given receiver or shot, $x$ refers to its distance along the spread, $y$ to its offset from the spread, and $z$ to its elevation. The coordinate system is right-handed, and its origin is arbitrary.

When editing the information file, be sure

1. To retain all labels such as "Spread name:," "Number of receivers =," etc.

2. To retain all blank lines.

3. That the number of receivers equals the number in the list of receivers.

4. That the number of shots equals the number in the list of shots.

\subsubsection{Seismic Data}

The seismic data must be in either the SEG-2 or the SU format (Section 5).

Program pick_sw executes properly only if two conditions on the data are met. First, two fields in the trace headers must be set: the number of samples per trace, and the sample interval. Second, traces must not have a time delay.

\subsubsection{File with Travel Times and Weights}

Frequently, re-examining the travel times and the weights is necessary. For this reason, program pick_sw always looks for the file with the travel times and the weights, whose name is specified in the information file (section 3.3.1). If the file exists, then it is read. Otherwise, the travel times are set to 0.0 , and the weights are set to 1.0.

The format of this file is described using "tt_picks.txt," which is and is an example of a travel time file and is located in directory test_data: 
Spread name: a1

\begin{tabular}{rrrrr} 
& Shots 101 \& 102 & \multicolumn{2}{c}{ Shots 103 \& 104} \\
Rec & Time & Weight & Time & Weight \\
101 & $1.52 e-002$ & 0.50 & $1.36 e-001$ & 1.00 \\
102 & $2.70 e-002$ & 0.50 & $1.35 e-001$ & 1.00 \\
103 & $3.60 e-002$ & 0.50 & $1.33 e-001$ & 1.00 \\
104 & $5.39 e-002$ & 1.00 & $1.31 e-001$ & 1.00 \\
105 & $6.55 e-002$ & 1.00 & $1.29 e-001$ & 1.00 \\
106 & $7.73 e-002$ & 1.00 & $1.27 e-001$ & 1.00 \\
107 & $8.99 e-002$ & 1.00 & $1.26 e-001$ & 1.00 \\
108 & $1.02 e-001$ & 1.00 & $1.24 e-001$ & 1.00 \\
109 & $1.09 e-001$ & 1.00 & $1.22 e-001$ & 1.00 \\
1010 & $1.11 e-001$ & 1.00 & $1.21 e-001$ & 1.00 \\
1011 & $1.12 e-001$ & 1.00 & $1.19 e-001$ & 1.00 \\
1012 & $1.14 e-001$ & 1.00 & $1.18 e-001$ & 1.00 \\
1013 & $1.15 e-001$ & 1.00 & $1.16 e-001$ & 1.00 \\
1014 & $1.17 e-001$ & 1.00 & $1.14 e-001$ & 1.00 \\
1015 & $1.19 e-001$ & 1.00 & $1.12 e-001$ & 1.00 \\
1016 & $1.22 e-001$ & 1.00 & $1.09 e-001$ & 1.00 \\
1017 & $1.24 e-001$ & 1.00 & $9.69 e-002$ & 1.00 \\
1018 & $1.26 e-001$ & 1.00 & $8.17 e-002$ & 1.00 \\
1019 & $1.27 e-001$ & 1.00 & $7.17 e-002$ & 1.00 \\
1020 & $1.29 e-001$ & 1.00 & $6.03 e-002$ & 1.00 \\
1021 & $1.31 e-001$ & 1.00 & $4.84 e-002$ & 0.50 \\
1022 & $1.32 e-001$ & 1.00 & $3.75 e-002$ & 0.25 \\
1023 & $1.34 e-001$ & 1.00 & $2.93 e-002$ & 0.25 \\
1024 & $1.36 e-001$ & 1.00 & $1.45 e-002$ & 0.25
\end{tabular}

Column 1 contains the receiver number. Columns 2 and 3 contain the travel times and the weights for shots 101 and 102. (The times are in seconds.) Likewise, columns 4 and 5 contain the travel times and the weights for shots 103 and 104.

\subsection{Execution}

To execute the program, there are four steps:

1. Start IDL.

2. From the menu, chose "File," and "Open Project." Open file "pick_sw.prj."

3. From the menu, chose "Project," "Compile," and "All Files."

4. From the menu, chose "Project," "Run."

The look and feel of the program is similar to that of most Windows programs, and so most users will readily figure-out how the progam works. If a question arises, users can get an answer in the "Help" documentation, which is loaded when the Help button is pressed. 
To exit from program pick_sw, select, on the main menu, "File" and then "Exit."

\section{Test Data}

In directory test_data are 9 files, which may be used to test whether program pick_sw is properly installed. The 9 files are described in sections 4.1 through 4.6.

\subsection{File "spr_info.txt": Information File}

This information file is setup for some $S$-wave refraction data, which were collected in an alluvial valley. The sediments consist mostly of sands and gravels and are about 4 meters thick. The bedrock is shale. The seismic spread consisted of 24 geophones and 4 shots. The 4 shots were at 2 different locations at which both polarities of the source were used. Details about the format of this file are in section 3.3.1.

\subsection{Files "17707.dat", "18707.dat", "19707.dat", "20707.dat": $S$-Wave Refraction Data}

These four files contain the seismic data stored in the SEG-2 format. For files "19707.dat" and "20707.dat", the shots were in the same location, but the polarities differed. Files "17707.dat" and "18707.dat" are similarly related.

Each file contains 24 traces, each of which consists of 2048 samples. The sample interval is 0.1 milli-seconds.

\subsection{File "tt_picks.txt": Travel Times}

Using program pick_sw, travel times were picked and weights were assigned to the picks. Both the times and the picks were written in file tt picks.txt. Details about the format of this file are in section 3.3.3.

\subsection{File "spread.ps": Plot of the Spread}

In the plot of the spread, each "X" represents two shots, which have the same location but opposite polarity. The labels adjacent to a cross are the shot numbers and the names of the files with the traces. Each dot represents a receiver (geophone). Approximately every 12 th receiver is labeled. The file is in postscript format.

This postscript file, as well as the postscript files described in sections 4.5 and 4.6, is easily converted to PDF files using Adobe Acrobat Distiller, and these files can be view with Adobe Acrobat Reader. In addition, these postscript files may be sent directly to a postscript-compatible printer.

\subsection{File "tt.ps": Plot of the Travel Times}


In the plot of the traveltimes, the vertical axis is the travel time, and the horizontal axis is the distance in the $\mathrm{x}$ direction. The filled circles represent travel times, the colors of the circles denote their weights, and the rectangles along the horizontal axis represent shot locations. The file is in postscript format.

\title{
4.6 File "traces.ps": Plot of the Traces for Shots 101 and 102
}

In the plot of the traces, the vertical axis represents time, and the horizontal axis represents distance in the $\mathrm{x}$ direction. For each receiver, there are two traces because there are two shots having the same location, but opposite polarity. The paired traces are plotted at the distance (in the $\mathrm{x}$ direction) of the associated receiver. The file is in postscript format.

\section{Further Information about the SEG-2 and SU Data Formats}

In 1990, members of the Society of Exploration Geophysicsts (SEG) established a standard format for storing seismic data that was collected for engineering-scale or smallscale investigations. This standard format is called "SEG-2" and is now widely used. Detailed information about this format is in an SEG report (Subcommittee of the SEG Engineering and Groundwater Geophysics Committee, 1990).

"SU" means Seismic Unix --- it is a software package for processing and displaying seismic data, and it runs on computers with the Unix operating system and X-windows (Cohen and Stockwell, 1999). The package is free and can be obtained from the anonymous ftp site at the Colorado School of Mines: ftp.cwp.mines.edu (138.67.12.4).

\section{Distributor of the IDL Programming Language}

IDL (Interactive Data Language) is designed especially for interactive visualization of scientific and technical data. To execute program pick_sw, version 5.5 or higher of IDL must be installed. IDL is not supplied in this Zip file; it may be obtained from

\author{
Research Systems, Inc. \\ 4990 East Pearl Circle \\ Boulder, Colorado 80301 \\ Telephone: 303.786 .9900 \\ Email: info@rsinc.com \\ Internet: http://www.rsinc.com
}

\section{Suggestions for Improving the Program}

If you have any suggestions for improving the program, please contact: 
Karl J. Ellefsen

U. S. Geological Survey

MS 964, Box 25046

Denver, CO 80225-0046

Email: ellefsen@usgs.gov

\section{Disclaimers}

This report has not been reviewed for conformity with U.S. Geological Survey editorial standards.

This open-file report was prepared by an agency of the United States Government. Neither the United States Government nor any agency thereof nor any of their employees makes any warranty, expressed or implied, or assumes any legal liability or responsibility for the accuracy, completeness, or usefulness of any information, apparatus, product, or process disclosed in this report or represents that its use would not infringe privately owned rights. Reference therein to any specific commercial product, process, or service by trade name, trademark, manufacturer, or otherwise does not constitute or imply its endorsement, recommendation, or favoring by the United States Government or any agency thereof.

Although all data and software in this open-file report have been used by the USGS, no warranty, expressed or implied, is made by the USGS as to the accuracy of the data and related materials and (or) the functioning of the software. The act of distribution shall not constitute any such warranty, and no responsibility is assumed by the USGS in the use of these data, software, or related materials.

\section{Acknowledgements}

This work was supported by two USGS projects: Sustainable Development of Industrial Minerals - Central Region Contribution to the National Industrial Minerals Project and Toxic Substances Hydrology Program.

\section{References}

Cohen, J. K., and Stockwell, Jr., J. W., 1999, CWP/SU: Seismic Unix release 33: A free package for seismic reseach and processing, Center for Wave Phenomenon, Colorado School of Mines.

Subcommittee of the SEG Engineering and Groundwater Geophysics Committee, Pullan, S. E., Chairman, 1990, Recommended standard for seismic (/radar) files in the personal computer environment:

Geophysics, vol. 55, no. 9, p. 1260-1271. 